\title{
EARTH ROTATION VELOCITY IN RELATION WITH DIFFERENT REFERENCE FRAMES
}

\author{
V. A. BRUMBERG \\ Institute of Applied Astronomy, \\ 197042 St.-Petersburg, Russia
}

\begin{abstract}
The high precision of present observations makes it reasonable to clear up a question about GRT (general relativity theory) corrections in the problem of Earth's rotation. The answer is that one may almost forget about GRT corrections when dealing in an adequate reference system (RS). The problem of Earth's rotation may be related to the relativistic hierarchy of RS started in (Brumberg and Kopejkin, 1989) and completed in (Klioner, 1993). Let letters B, G and T be related to barycentric, geocentric and topocentric RS, respectively. Let DRS and KRS be dynamically nonrotating or kinematically nonrotating RS, respectively. From the dynamical equations of rotation it follows that the most adequate system for studying the Earth's rotation is DGRS. Apart from the geophysical factors the rotation of the Earth in this system is fairly well approximated by the rigid-body rotation with some angular velocity $\hat{\omega}^{i}$. The same rotation of the Earth as considered in BRS and DTRS may be also approximated by the rigid-body rotation but with some additive relativistic corrections and with other angular velocities $\omega^{i}$ and $\tilde{\omega}^{i}$, respectively. Substituting these three rotation relations into four-dimensional BRS-DGRS and DGRS-DTRS transformations one may express $\omega^{i}$ and $\tilde{\omega}^{i}$ in terms of $\hat{\omega}^{i}$ and determine the additive relativistic corrections in BRS and BTRS. These corrections are of importance for treating kinematics problems in various coordinate systems and for obtaining physically meaningful solutions of the dynamical equations of rotation in the barycentric reference system.
\end{abstract}

The complete text will be published in Journal of Geodynamics.

\section{References}

Brumberg V.A. and Kopejkin S.M., 1989, in: Kovalevsky J., Mueller I.I., Kolaczek B. (eds.) Reference Frames. Kluwer, Dordrecht, p. 115

Klioner S.A., 1993, A\&A 279, 273 